\title{
A special case of Vinogradov's mean value theorem
}

\author{
by
}

\author{
R. C. Vaughan (London) and T. D. Wooley (Ann Arbor, Mich.)
}

In honorem J. W. S. Cassels annos LXXV nati

1. Introduction. In analytic number theory, estimates for the number, $J_{s, k}(P)$, of solutions of the system of equations

$$
\sum_{i=1}^{s}\left(x_{i}^{j}-y_{i}^{j}\right)=0 \quad(1 \leq j \leq k)
$$

with $x_{i}, y_{i} \in[1, P] \cap \mathbb{Z}$ are of great utility. This is perhaps best illustrated by the seminal works of Vinogradov from the first half of this century (see, for example, $[1,6]$ ). Despite modern developments, such estimates remain the primary tool in establishing the best known results concerning the zerofree region of the Riemann zeta function, and the smallest number $\widetilde{G}(k)$ of variables for which the asymptotic formula holds in Waring's problem. When $s<\frac{1}{2} k(k+1)$ and $P$ is large compared to $s$, it is widely conjectured that $J_{s, k}(P) \sim s ! P^{s}$. This is an immediate consequence of Newton's formulae on the powers of the roots of a polynomial when $1 \leq s \leq k$, but when $s>k+1$ the latter asymptotic formula seems far beyond the grasp of current technology. Our primary purpose in this memoir is to establish in a rather sharp form the desired asymptotic formula in the case $s=k+1$.

When $s$ is a natural number, let $T_{s}(P)$ denote the number of $s$-tuples $\mathbf{x}$ and $\mathbf{y}$ in which $1 \leq x_{i}, y_{i} \leq P(1 \leq i \leq s)$, and the $x_{i}$ are a permutation of the $y_{j}$, so that in particular, $T_{s}(P)=s ! P^{s}+O_{s}\left(P^{s-1}\right)$. In Section 2 we establish the strong form below of the asymptotic formula $J_{k+1, k}(P) \sim$ $T_{k+1}(P)$, and in connection with this we define

$$
\alpha_{n}=\min _{\substack{1 \leq r \leq n \\ r \in \mathbb{N}}}(r+n / r) .
$$

Research of the first author supported by an EPSRC Senior Fellowship.

Research of the second author supported by NSF grant DMS-9303505 and a Fellowship from the David and Lucile Packard Foundation. 
THEOREM 1 . When $k \geq 3$,

$$
J_{k+1, k}(P)-T_{k+1}(P) \ll_{\varepsilon, k} P^{\alpha_{k+1}+\varepsilon},
$$

and consequently,

$$
J_{k+1, k}(P)=T_{k+1}(P)+O_{k}\left(P^{\sqrt{4 k+5}}\right) .
$$

For comparison, Hua [3, Lemma 5.4] provides the upper bound $J_{k+1, k}(P)$ $\ll_{k} P^{k+1}(\log 2 P)^{2^{k}-1}$, and very recently Vaughan and Wooley [5, Theorem 1.4] have obtained the bound (1.3) with $\alpha_{k+1}$ replaced by $\frac{1}{2}(k+5)$. The upper bound (1.3) is non-trivial for $k \geq 4$, and is superior to those obtained hitherto for $k \geq 6$. The methods developed here are susceptible to further small improvements, but for larger $k$ they are of no great significance. However, it is possible to obtain (1.3) with the exponent $\alpha_{k+1}$ replaced by $33 / 8$ and $23 / 5$ when $k=4$ and $k=5$ respectively. We briefly outline this refinement at the end of Section 2 .

For the sake of completeness we remark that in the cases $k=2,3$, Rogovskaya [4] and Vaughan and Wooley [5, Theorem 1.5], respectively, have established the estimates

$$
J_{3,2}(P)=\frac{18}{\pi^{2}} P^{3} \log P+O\left(P^{3}\right),
$$

and, when $P$ is large,

$$
P^{2} \log P \ll J_{4,3}(P)-T_{4}(P) \ll P^{10 / 3}(\log 2 P)^{35} .
$$

We note that the strength of the upper bound (1.3) is sufficient for applications to quasi-diagonal behaviour in the context of Vinogradov's mean value theorem (see [7, Lemmata 2.2 and 4.2] for details).

It seems worth remarking that when $P$ is large, the existence of one nontrivial solution, $\mathbf{x}, \mathbf{y}$, of the system (1.1) implies the existence of $\gg_{\mathbf{x}, \mathbf{y}} P^{2}$ non-trivial solutions $\mathbf{x}^{\prime}, \mathbf{y}^{\prime}$ with $1 \leq x_{i}^{\prime}, y_{i}^{\prime} \leq P(1 \leq i \leq s)$. This follows by taking

$$
\mathbf{x}^{\prime}=q \mathbf{x}+r \quad \text { and } \quad \mathbf{y}^{\prime}=q \mathbf{y}+r,
$$

with $1 \leq q<P / \max \left\{x_{i}, y_{i}\right\}$ and $1 \leq r \leq P-q \max \left\{x_{i}, y_{i}\right\}$. Thus whenever $J_{s, k}(Q)-T_{s}(Q)>0$ and $P \geq Q$, one has $J_{s, k}(P)-T_{s}(P) \gg_{k} P^{2}$. The current state of knowledge concerning the problem of Prouhet and Tarry (see Theorem 411 and the note on page 339 of [2]) therefore suffices to demonstrate that when $1 \leq k \leq 9$ and $P$ is large, one has $J_{k+1, k}(P)-$ $T_{k+1}(P) \gg_{k} P^{2}$. Whether or not there exist non-trivial solutions of the system (1.1) when $s=k+1$ and $k>9$ remains open to speculation.

Denote by $S_{k}(P)$ the number of solutions of the system

$$
\sum_{i=1}^{k}\left(x_{i}^{j}-y_{i}^{j}\right)=0 \quad(j=1,2, \ldots, k-2 \text { and } k),
$$


with $x_{i}, y_{i} \in[1, P] \cap \mathbb{Z}(1 \leq i \leq k)$. Similarities in the underlying algebraic structure enable us in Section 3 to adapt our methods successfully in order to estimate $S_{k}(P)-T_{k}(P)$.

THEOREM 2. When $k \geq 3$,

$$
S_{k}(P)-T_{k}(P) \ll_{\varepsilon, k} P^{\alpha_{k}+\varepsilon},
$$

and consequently,

$$
S_{k}(P)=T_{k}(P)+O_{k}\left(P^{\sqrt{4 k+1}}\right) .
$$

In this situation, Hua [3, Lemma 5.2] provides the upper bound $S_{k}(P)$ $\ll_{k} P^{k}(\log 2 P)^{k\left(2^{k-1}-1\right)}$, and very recently Vaughan and Wooley [5, Theorem 1.3] have obtained the bound (1.6) with $\alpha_{k}$ replaced by $\frac{1}{2}(k+3)$. When $k$ is large the superiority of (1.6) over the latter estimates is amply illuminated by (1.7). For the sake of completeness we remark that when $k=3$ and $P$ is large, Vaughan and Wooley [5, Theorem 1.2] have established the estimate

$$
P^{2}(\log P)^{5} \ll S_{3}(P)-6 P^{3} \ll P^{2}(\log P)^{5} .
$$

Our proof of Theorem 1 in Section 2 is elementary, and forms a natural extension to that used in [5, Section 9]. We use polynomial identities to bound the number of solutions of the system (1.1) counted by $J_{k+1, k}(P)-T_{k+1}(P)$ in terms of the number of solutions of a linear system subject to multiplicative constraints. The latter constraints lead, via extraction of common factors, to a system amenable to linear algebra and divisor function estimates. For smaller $k$ one may refine the estimate (1.3) somewhat by better exploiting certain of the auxiliary variables which arise in our argument. We briefly sketch at the end of Section 2 how such refinements may be established. By a fortunate coincidence, a very similar system also arises through the use of polynomial identities in the treatment of the system (1.5), and thus in Section 3 we are able to establish Theorem 2 through a similar argument.

Throughout, « and 》 denote Vinogradov's well-known notation. Implicit constants in both the notations of Vinogradov and Landau will depend at most on $\varepsilon, k$ and $r$. For the sake of concision, we make frequent use of vector notation. Thus, for example, we abbreviate $\left(c_{1}, \ldots, c_{t}\right)$ to c. Finally, we write $\left(a_{1}, \ldots, a_{s}\right)$ for the greatest common divisor of $a_{1}, \ldots, a_{s}$, and we have been careful to ensure that any possible ambiguity can be resolved by the context.

2. The proof of Theorem 1. Let $U_{k}(P)$ denote the number of solutions of the system

$$
\sum_{i=1}^{k+1}\left(x_{i}^{j}-y_{i}^{j}\right)=0 \quad(1 \leq j \leq k)
$$


with $1 \leq x_{i}, y_{i} \leq P(1 \leq i \leq k+1)$, and satisfying the condition that $\left(x_{1}, \ldots, x_{k+1}\right)$ is not a permutation of $\left(y_{1}, \ldots, y_{k+1}\right)$. In this section we establish the estimate

$$
U_{k}(P) \ll P^{\alpha_{k+1}+\varepsilon},
$$

from which the main conclusion of Theorem 1 follows immediately. Meanwhile, (1.4) follows by taking $r$ to be the integer closest to $\sqrt{k+1}$ in the formula for $\alpha_{k+1}$, and then applying some mundane analysis.

We start by observing that the polynomial $p(\xi ; \mathbf{z})$, defined by

$$
p(\xi ; \mathbf{z})=\prod_{i=1}^{k+1}\left(z_{i}-\xi\right)-\prod_{j=1}^{k+1} z_{j},
$$

considered as a polynomial in $\xi$, has coefficients which are symmetric polynomials in $z_{1}, \ldots, z_{k+1}$ of degree at most $k$. Thus for each solution $\mathbf{x}, \mathbf{y}$ of the system $(2.1)$ counted by $U_{k}(P)$, one has $p(\xi ; \mathbf{x})=p(\xi ; \mathbf{y})$. Consequently, for each $s$ with $1 \leq s \leq k+1$,

$$
\prod_{j=1}^{k+1}\left(y_{j}-x_{s}\right)=y_{1} \ldots y_{k+1}-x_{1} \ldots x_{k+1}
$$

whence

$$
\prod_{i=1}^{k+1}\left(y_{i}-x_{s}\right)=\prod_{j=1}^{k+1}\left(y_{j}-x_{t}\right) \quad(1 \leq s<t \leq k+1) .
$$

Further, if $x_{i}=y_{j}$ for any $i$ and $j$, then the equation (2.3) with $s=i$ implies that $x_{1} \ldots x_{k+1}=y_{1} \ldots y_{k+1}$. In combination with the equations (2.1), therefore, the use of elementary properties of symmetric polynomials leads to the conclusion that $\left(x_{1}, \ldots, x_{k+1}\right)$ is a permutation of $\left(y_{1}, \ldots, y_{k+1}\right)$, contradicting the assumption that $\mathbf{x}, \mathbf{y}$ is a solution counted by $U_{k}(P)$. We may thus suppose that $x_{i}=y_{j}$ for no $i$ and $j$.

We divide the solutions $\mathbf{x}, \mathbf{y}$ of $(2.1)$ counted by $U_{k}(P)$ into two types according to an integer parameter $r$ with $1<r \leq k+1$. Let $V_{1, r}(P)$ denote the number of such solutions in which there are fewer than $r$ distinct values amongst the $x_{i}$, and let $V_{2, r}(P)$ denote the corresponding number of solutions in which there are at least $r$ distinct values amongst the $x_{i}$. Then

$$
U_{k}(P)=V_{1, r}(P)+V_{2, r}(P) .
$$

Consider first the solutions counted by $V_{1, r}(P)$. Fix any one of the $O\left(P^{r-1}\right)$ possible choices for $\mathbf{x}$, and fix also one of the $O(P)$ available choices for $y_{1}$. By interchanging the rôles of $\mathbf{x}$ and $\mathbf{y}$ in (2.4), we obtain

$$
\prod_{i=1}^{k+1}\left(x_{i}-y_{s}\right)=\prod_{j=1}^{k+1}\left(x_{j}-y_{1}\right) \quad(1 \leq s \leq k+1) .
$$


Thus, since each of the integers $x_{j}-y_{1}$ is fixed, when $2 \leq s \leq k+1$ each $y_{s}$ is determined by a non-trivial polynomial. Consequently, there are $O(1)$ possible choices for $y_{2}, \ldots, y_{k+1}$, whence

$$
V_{1, r}(P) \ll P^{r} .
$$

Next consider a solution $\mathbf{x}, \mathbf{y}$ counted by $V_{2, r}(P)$. By relabelling variables we may suppose that $x_{1}, \ldots, x_{r}$ are distinct. Suppose temporarily that the integers $y_{1}$ and $y_{i}-x_{s}(1 \leq i \leq k+1,1 \leq s \leq r)$ are determined. Then plainly $x_{s}$ is determined for $1 \leq s \leq r$, whence $y_{i}$ is determined for $1 \leq i \leq$ $k+1$. Moreover, when $r<s \leq k+1$, the integers $x_{s}$ may be determined from the polynomial equations (2.4) with $t=1$. Then since there are $O(P)$ possible choices for $y_{1}$, we may conclude that given $y_{i}-x_{s}(1 \leq i \leq k+1,1 \leq$ $s \leq r)$, there are $O(P)$ possible choices for $\mathbf{x}, \mathbf{y}$. Substituting $u_{0 j}=x_{j}-y_{1}$ and $u_{i j}=y_{i+1}-x_{j}(1 \leq i \leq k, 1 \leq j \leq r)$, we deduce from (2.4)-(2.6) that

$$
U_{k}(P) \ll P W_{r}(P)+P^{r},
$$

where $W_{r}(P)$ denotes the number of solutions of the system

$$
\prod_{i_{1}=0}^{k} u_{i_{1} 1}=\prod_{i_{2}=0}^{k} u_{i_{2} 2}=\ldots=\prod_{i_{r}=0}^{k} u_{i_{r} r}
$$

with

$$
u_{01}+u_{i 1}=u_{02}+u_{i 2}=\ldots=u_{0 r}+u_{i r} \quad(1 \leq i \leq k)
$$

and

$$
1 \leq\left|u_{i j}\right| \leq P \quad(0 \leq i \leq k, 1 \leq j \leq r),
$$

and with the $u_{0 j}$ distinct for $1 \leq j \leq r$.

We now use the equations (2.8) to eliminate common factors amongst the $u_{i j}$. In order to make our description of this process precise, we record some notational devices. Let $\mathcal{I}$ denote the set of indices $\mathbf{i}=\left(i_{1}, \ldots, i_{r}\right)$ with $0 \leq i_{m} \leq k(1 \leq m \leq r)$. Define a map $\phi: \mathcal{I} \rightarrow\left[0,(k+1)^{r}\right) \cap \mathbb{Z}$ by

$$
\phi(\mathbf{i})=\sum_{m=1}^{r} i_{m}(k+1)^{m-1} .
$$

Then $\phi$ is bijective, and we can define the successor, $\mathbf{i}+1$, of the index $\mathbf{i}$ by

$$
\mathbf{i}+1=\phi^{-1}(\phi(\mathbf{i})+1) .
$$

When $h \in \mathbb{N}$, we define $\mathbf{i}+h$ inductively by $\mathbf{i}+(h+1)=(\mathbf{i}+h)+1$. Further, when $\mathbf{i} \in \mathcal{I}$, we write $\mathcal{J}(\mathbf{i})$ for the set of $\mathbf{j} \in \mathcal{I}$ such that for some $h \in \mathbb{N}$ one has $\mathbf{j}+h=\mathbf{i}$. We now define the integers $\alpha_{\mathbf{i}}$, with $\mathbf{i} \in \mathcal{I}$, as follows. We put $\alpha_{\mathbf{0}}=\left(u_{01}, u_{02}, \ldots, u_{0 r}\right)$, and suppose at stage $\mathbf{i}$ that $\alpha_{\mathbf{j}}$ has been defined for 
$\mathbf{j} \in \mathcal{J}(\mathbf{i})$. We then define $\alpha_{\mathbf{i}}$ by

$$
\alpha_{\mathbf{i}}=\left(\frac{u_{i_{1} 1}}{\beta_{\mathbf{i}}^{(1)}}, \frac{u_{i_{2} 2}}{\beta_{\mathbf{i}}^{(2)}}, \ldots, \frac{u_{i_{r} r}}{\beta_{\mathbf{i}}^{(r)}}\right), \quad \text { where } \quad \beta_{\mathbf{i}}^{(m)}=\prod_{\substack{\mathbf{j} \in \mathcal{J}(\mathbf{i}) \\ j_{m}=i_{m}}} \alpha_{\mathbf{j}},
$$

and here we adopt the convention that the empty product is unity. It follows that when $0 \leq l \leq k$ and $1 \leq m \leq r$, one has

$$
u_{l m}=\prod_{\substack{\mathbf{j} \in \mathcal{I} \\ j_{m}=l}} \alpha_{\mathbf{j}}
$$

We now consider $\alpha_{\mathbf{i}}$, with $\mathbf{i} \in \mathcal{I}$, as variables, and for the sake of transparency write

$$
\widetilde{\alpha}_{l m}=\prod_{\substack{\mathbf{j} \in \mathcal{I} \\ j_{m}=l}} \alpha_{\mathbf{j}}
$$

Then it follows from the discussion of the preceding paragraph that $W_{r}(P) \leq$ $X_{r}(P)$, where $X_{r}(P)$ denotes the number of solutions of the system

$$
\widetilde{\alpha}_{01}+\widetilde{\alpha}_{i 1}=\widetilde{\alpha}_{02}+\widetilde{\alpha}_{i 2}=\ldots=\widetilde{\alpha}_{0 r}+\widetilde{\alpha}_{i r} \quad(1 \leq i \leq k),
$$

with the $\widetilde{\alpha}_{0 j}$ distinct for $1 \leq j \leq r$, and with

$$
1 \leq\left|\widetilde{\alpha}_{i j}\right| \leq P \quad(0 \leq i \leq k, 1 \leq j \leq r)
$$

Thus by (2.7),

$$
U_{k}(P) \ll P X_{r}(P)+P^{r} .
$$

Having eliminated the multiplicative conditions inherent in our system, we are left to investigate the system (2.13). When $1 \leq p \leq r$, we write

$$
A_{p}=\prod_{\substack{\mathbf{i} \in \mathcal{I} \\ i_{l}>i_{p}(l \neq p)}} \alpha_{\mathbf{i}}
$$

It follows easily that

$$
\left|\prod_{p=1}^{r} A_{p}\right| \leq \prod_{\mathbf{i} \in \mathcal{I}}\left|\alpha_{\mathbf{i}}\right| \leq P^{k+1},
$$

and thus in any solution $\boldsymbol{\alpha}$ counted by $X_{r}(P)$, there exists a $p$ with $1 \leq p \leq r$ such that $\left|A_{p}\right| \leq P^{(k+1) / r}$. Moreover, given $l$ with $1 \leq l \leq r$, it follows from (2.13) and (2.14) that for each solution $\boldsymbol{\alpha}$ counted by $X_{r}(P)$, there exist integers $L_{j}$ with $0<\left|L_{j}\right| \leq 2 P$ such that when $1 \leq j \leq r$ and $j \neq l$,

$$
\widetilde{\alpha}_{0 l}-\widetilde{\alpha}_{0 j}=-L_{j}, \quad \widetilde{\alpha}_{i l}-\widetilde{\alpha}_{i j}=L_{j} \quad(1 \leq i \leq k) .
$$


By relabelling variables, therefore, we deduce that $X_{r}(P) \ll Y_{r}(P)$, where $Y_{r}(P)$ denotes the number of solutions of the system

$$
\widetilde{\alpha}_{01}-\widetilde{\alpha}_{0 j}=-L_{j}, \quad \widetilde{\alpha}_{i 1}-\widetilde{\alpha}_{i j}=L_{j} \quad(2 \leq j \leq r, 1 \leq i \leq k),
$$

with

$$
1 \leq\left|L_{j}\right| \leq 2 P \quad(2 \leq j \leq r),
$$

and with the $\alpha_{\mathbf{i}}$ satisfying (2.14) and the inequality

$$
\left|A_{1}\right| \leq P^{(k+1) / r}
$$

where $A_{1}$ is defined by (2.16). Further, by (2.15),

$$
U_{k}(P) \ll P Y_{r}(P)+P^{r} .
$$

We claim that when the variables $L_{2}, \ldots, L_{r}$, and $\alpha_{\mathbf{i}}$ with

$$
\mathbf{i} \in \mathcal{I} \quad \text { and } \quad i_{l}>i_{1} \quad(2 \leq l \leq r),
$$

are fixed, then there are $O\left(P^{\varepsilon}\right)$ possible choices for the $\alpha_{\mathbf{i}}$ satisfying (2.14) and (2.17). If such is the case, then by combining (2.18)-(2.20) with standard estimates for the divisor function, we obtain $U_{k}(P) \ll P^{r+(k+1) / r+\varepsilon}$, and so the main conclusion of Theorem 1 follows.

It remains to establish the latter proposition, which we prove inductively as follows. For a fixed choice of the $\alpha_{\mathbf{i}}$ with $\mathbf{i}$ satisfying (2.21), we suppose at step $t$ that there are $O\left(P^{t \varepsilon}\right)$ possible choices for those variables $\alpha_{\mathbf{i}}$ for which i satisfies the condition that $i_{l}<t$ for some $l$ with $1 \leq l \leq r$. Observe first that (2.17) implies that

$$
\widetilde{\alpha}_{0 j}=\widetilde{\alpha}_{01}+L_{j} \quad(2 \leq j \leq r) .
$$

We have supposed, moreover, that $L_{2}, \ldots, L_{r}$ are fixed and non-zero, and that the variables $\alpha_{\mathbf{i}}$ for which $i_{1}=0$ and $i_{l}>0(2 \leq l \leq r)$, are also fixed. Then by using standard estimates for the divisor function, it follows from (2.22) that there are $O\left(P^{\varepsilon}\right)$ possible choices for the $\alpha_{\mathbf{i}}$ for which $\mathbf{i}$ satisfies the condition that $i_{l}=0$ for some $l$ with $1 \leq l \leq r$. Thus our hypothesis holds when $t=1$.

Suppose next that the hypothesis is satisfied for a $t \geq 1$, and consider a fixed one of the $O\left(P^{t \varepsilon}\right)$ possible choices for the $\alpha_{\mathbf{i}}$ for which $i_{l}<t$ for some $l$ with $1 \leq l \leq r$. It follows from (2.17) that

$$
\widetilde{\alpha}_{t j}=\widetilde{\alpha}_{t 1}-L_{j} \quad(2 \leq j \leq r) .
$$

Once again, $L_{2}, \ldots, L_{r}$ are fixed and non-zero. Moreover, if

$$
i_{1}=t \quad \text { and } \quad i_{l} \neq t \quad(2 \leq l \leq r),
$$

then either some $i_{l}<t$, or else $i_{l}>t(2 \leq l \leq r)$, and thus the variables $\alpha_{\mathbf{i}}$ for which $\mathbf{i}$ satisfies (2.24) may also be supposed fixed. Then by using standard estimates for the divisor function, it follows from (2.23) that there are $O\left(P^{\varepsilon}\right)$ 
possible choices for the variables $\alpha_{\mathbf{i}}$ for which $\mathbf{i}$ satisfies the condition that $i_{l}=t$ for some $l$ with $1 \leq l \leq r$. Consequently, there are $O\left(P^{(t+1) \varepsilon}\right)$ possible choices for the variables $\alpha_{\mathbf{i}}$ for which $\mathbf{i}$ satisfies the condition that $i_{l} \leq t$ for some $l$ with $1 \leq l \leq r$, and so the inductive hypothesis holds with $t$ replaced by $t+1$. This completes the induction, and the proof of the main conclusion of Theorem 1.

By better exploiting the variables $\alpha_{\mathbf{i}}$ not occurring as factors of the $A_{p}$, it is possible to improve the upper bound (1.3) a little. Although for large $k$ these improvements are not of great significance, for smaller $k$ they may be of some interest. We sketch below one possible approach to obtaining such refinements.

We start by making an observation concerning the solutions counted by $X_{r}(P)$. Let $\mathcal{I}^{+}$denote the set of indices $\mathbf{i} \in \mathcal{I}$ such that $i_{l}>0(1 \leq l \leq r)$, and let $\mathcal{I}^{*}$ denote the corresponding set of indices subject to the additional condition that for some $p$ with $1 \leq p \leq r$, one has $i_{l}>i_{p}$ whenever $l \neq p$. $\operatorname{Thus} \operatorname{card}\left(\mathcal{I}^{+}\right)=k^{r}$, and $\operatorname{card}\left(\mathcal{I}^{*}\right)=r \psi(k)$, where

$$
\psi(k)=\sum_{i=1}^{k-1} i^{r-1}<k^{r} / r .
$$

Observe that by considering changes of variables corresponding to permuting the indices $i_{l}$, for each fixed $l$, it follows with little difficulty from the argument of the proof of Theorem 1 that $W_{r}(P) \ll X_{r}(P)$, where $X_{r}(P)$ is defined as before, but now one may impose the additional condition

$$
\prod_{\mathbf{i} \in \mathcal{I}^{*}}\left|\alpha_{\mathbf{i}}\right| \leq\left(\prod_{\mathbf{i} \in \mathcal{I}^{+}}\left|\alpha_{\mathbf{i}}\right|\right)^{\operatorname{card}\left(\mathcal{I}^{*}\right) / \operatorname{card}\left(\mathcal{I}^{+}\right)} .
$$

It follows that

$$
\begin{aligned}
\left|\prod_{p=1}^{r} A_{p}\right| & \leq\left(\prod_{p=1}^{r} \prod_{\substack{\mathbf{i} \in \mathcal{I} \\
i_{p}=0 \\
i_{l}>0(l \neq p)}}\left|\alpha_{\mathbf{i}}\right|\right)\left(\prod_{\mathbf{i} \in \mathcal{I}^{*}}\left|\alpha_{\mathbf{i}}\right|\right) \\
& \leq\left(\prod_{p=1}^{r} \prod_{\substack{\mathbf{i} \in \mathcal{I} \\
i_{p}=0}}\left|\alpha_{\mathbf{i}}\right|\right)^{1-r \psi(k) / k^{r}}\left(\prod_{\mathbf{i} \in \mathcal{I}}\left|\alpha_{\mathbf{i}}\right|\right)^{r \psi(k) / k^{r}} \\
& \leq\left(P^{r}\right)^{1-r \psi(k) / k^{r}}\left(P^{k+1}\right)^{r \psi(k) / k^{r}} .
\end{aligned}
$$

Consequently, in any solution $\boldsymbol{\alpha}$ counted by $X_{r}(P)$, there exists a $p$ with $1 \leq p \leq r$ such that

$$
\left|A_{p}\right| \leq P^{1+(k+1-r) \psi(k) / k^{r}} .
$$

We may now prosecute the same argument as before, but now delivering the 
conclusion

$$
U_{k}(P) \ll P^{\beta_{k}+\varepsilon},
$$

where

$$
\beta_{k}=\min _{\substack{2 \leq r \leq k+1 \\ r \in \mathbb{N}}}\left(r+1+\frac{k+1-r}{k^{r}} \sum_{i=1}^{k-1} i^{r-1}\right) .
$$

When $r=2$, the expression on the right-hand side of (2.25) yields

$$
\beta_{k} \leq \frac{1}{2}(k+4+1 / k) .
$$

Thus when $k=4$, and when $k=5$, this refined argument with $r=2$ yields the sharpest bounds available to us, namely

$$
U_{4}(P) \ll P^{33 / 8+\varepsilon} \quad \text { and } \quad U_{5}(P) \ll P^{23 / 5+\varepsilon} \text {. }
$$

3. The proof of Theorem 2. Having illustrated our method in Section 2 we can afford to be brief in our proof of Theorem 2 . We start by recording an observation from [5, Section 8]. From [5, (8.24)], together with the equation obtained by reversing the rôles of $\mathbf{x}$ and $\mathbf{y}$ in that equation, it follows that

$$
S_{k}(P)-T_{k}(P) \ll R_{k}(k P),
$$

where $R_{k}(Q)$ denotes the number of solutions of the system

$$
\begin{array}{ll}
x_{v} \prod_{i=1}^{k}\left(y_{i}-x_{u}\right)=x_{u} \prod_{j=1}^{k}\left(y_{j}-x_{v}\right) & (1 \leq u<v \leq k), \\
y_{v} \prod_{i=1}^{k}\left(x_{i}-y_{u}\right)=y_{u} \prod_{j=1}^{k}\left(x_{j}-y_{v}\right) & (1 \leq u<v \leq k),
\end{array}
$$

with $1 \leq x_{i}, y_{i} \leq Q(1 \leq i \leq k)$, and satisfying the condition that $x_{i}=y_{j}$ for no $i$ and $j$.

We divide the solutions $\mathbf{x}, \mathbf{y}$ of (3.2) and (3.3) counted by $R_{k}(Q)$ into two types according to an integer parameter $r$ with $1<r \leq k$. Let $N_{1, r}(Q)$ denote the number of such solutions in which there are fewer than $r$ distinct values amongst the $x_{i}$, and let $N_{2, r}(Q)$ denote the corresponding number of solutions in which there are at least $r$ distinct values amongst the $x_{i}$. Then

$$
R_{k}(Q)=N_{1, r}(Q)+N_{2, r}(Q) .
$$

Consider first the solutions counted by $N_{1, r}(Q)$. Fix any one of the $O\left(Q^{r-1}\right)$ possible choices for $\mathbf{x}$, and fix also any one of the $O(Q)$ possible choices for $y_{1}$. Then since each of the integers $x_{j}-y_{1}(1 \leq j \leq k)$ is fixed, when $2 \leq u \leq k$ each $y_{u}$ is determined by the non-trivial polynomial 
equation (3.3) with $v=1$. Consequently, there are $O(1)$ possible choices for $y_{2}, \ldots, y_{k}$, whence

$$
N_{1, r}(Q) \ll Q^{r} \text {. }
$$

Next consider a solution $\mathbf{x}, \mathbf{y}$ counted by $N_{2, r}(Q)$. By relabelling variables we may suppose that $x_{1}, \ldots, x_{r}$ are distinct. Suppose temporarily that the integers $x_{u}$ and $y_{i}-x_{u}(1 \leq i \leq k, 1 \leq u \leq r)$ are determined. Then plainly $x_{u}$ and $y_{i}$ are determined for $1 \leq i \leq k$ and $1 \leq u \leq r$. Moreover, when $r<u \leq k$, the integers $x_{u}$ may be determined from the polynomial equations (3.2) with $v=1$. Then since there are $O\left(Q^{r}\right)$ possible choices for $x_{1}, \ldots, x_{r}$, we may conclude that given $y_{i}-x_{u}(1 \leq i \leq k, 1 \leq u \leq r)$, there are $O\left(Q^{r}\right)$ possible choices for $\mathbf{x}, \mathbf{y}$. Substituting $u_{i j}=y_{i}-x_{j}(1 \leq$ $i \leq k, 1 \leq j \leq r)$, we deduce from (3.2)-(3.5) that

$$
R_{k}(Q) \ll Q^{r} \max _{\mathbf{x}} M_{r}(Q ; \mathbf{x})+Q^{r},
$$

where the maximum is taken over $x_{1}, \ldots, x_{r}$ with

$$
1 \leq x_{i} \leq Q \quad(1 \leq i \leq r)
$$

and with the $x_{i}$ distinct, and where $M_{r}(Q ; \mathbf{x})$ denotes the number of solutions of the system (2.8) with

$$
\begin{gathered}
x_{1}+u_{i 1}=x_{2}+u_{i 2}=\ldots=x_{r}+u_{i r} \quad(1 \leq i \leq k), \\
1 \leq\left|u_{i j}\right| \leq Q \quad(1 \leq i \leq k, 1 \leq j \leq r),
\end{gathered}
$$

and

$$
u_{0 i}=x_{i}^{-1} \prod_{j=1}^{r} x_{j} \quad(1 \leq i \leq r) .
$$

We may now extract common factors between the variables $u_{i j}$ precisely as in Section 2. Thus, on recalling the notation of Section 2, we deduce that there are integers $\alpha_{\mathbf{i}}(\mathbf{i} \in \mathcal{I})$ such that when $0 \leq l \leq k$ and $1 \leq m \leq r$, one has (2.11). We note that in view of (3.8), the $u_{0 i}$ are fixed. Thus, by making use of standard estimates for the divisor function, we deduce that there are $O\left(Q^{\varepsilon}\right)$ possible choices for the $\alpha_{\mathbf{j}}$ for which $j_{m}=0$ for some $m$ with $1 \leq m \leq r$. Treating the $\alpha_{\mathbf{i}}$ now as variables, and recalling the notation (2.12), we conclude that $M_{r}(Q ; \mathbf{x}) \ll Q^{\varepsilon} K_{r}(Q ; \mathbf{x})$, where $K_{r}(Q ; \mathbf{x})$ denotes the number of solutions of the system

$$
x_{1}+\widetilde{\alpha}_{i 1}=x_{2}+\widetilde{\alpha}_{i 2}=\ldots=x_{r}+\widetilde{\alpha}_{i r} \quad(1 \leq i \leq k),
$$

with

$$
1 \leq\left|\widetilde{\alpha}_{i j}\right| \leq Q \quad(1 \leq i \leq k, 1 \leq j \leq r)
$$

and with the variables $\alpha_{\mathbf{i}}$, for which $i_{m}=0$ for some $m$ with $1 \leq m \leq r$, fixed. 
We investigate the system (3.9) following the trail laid down in Section 2. When $1 \leq p \leq r$, we write $B_{p}=\prod_{\mathbf{i}}^{*} \alpha_{\mathbf{i}}$, where the product is over $\mathbf{i} \in \mathcal{I}$ for which $i_{l}>i_{p}(l \neq p)$, and $i_{l}>0(1 \leq l \leq r)$. It follows that

$$
\left|\prod_{p=1}^{r} B_{p}\right| \leq \prod_{\substack{\left.\mathbf{i} \in \mathcal{I} \\ i_{l}>l \leq l \leq r\right)}}\left|\alpha_{\mathbf{i}}\right| \leq Q^{k}
$$

and thus in any solution $\boldsymbol{\alpha}$ counted by $K_{r}(Q ; \mathbf{x})$, there exists a $p$ with $1 \leq p \leq r$ such that $\left|B_{p}\right| \leq Q^{k / r}$. By relabelling variables, we therefore deduce that

$$
K_{r}(Q ; \mathbf{x}) \ll I_{r}(Q ; \mathbf{x}),
$$

where $I_{r}(Q ; \mathbf{x})$ denotes the number of solutions of the system

$$
\widetilde{\alpha}_{i 1}-\widetilde{\alpha}_{i j}=L_{j} \quad(2 \leq j \leq r, 1 \leq i \leq k),
$$

with $L_{j}=x_{j}-x_{1}(2 \leq j \leq r)$, and with the $\alpha_{\mathbf{i}}$ satisfying (3.10) and the inequality

$$
\left|B_{1}\right| \leq Q^{k / r}
$$

We claim that when the variables $\alpha_{\mathbf{i}}$, with $\mathbf{i}$ satisfying (2.21), are fixed, then there are $O\left(Q^{\varepsilon}\right)$ possible choices for the $\alpha_{\mathbf{i}}$ satisfying (3.10) and (3.11). If such is the case, then by combining (3.12) with standard estimates for the divisor function, we obtain $I_{r}(Q ; \mathbf{x}) \ll Q^{k / r+\varepsilon}$, whence by (3.6) we have $R_{k}(Q) \ll Q^{r+k / r+\varepsilon}$. The main conclusion of Theorem 2 follows immediately.

But the claimed conclusion may be established precisely as in the argument of the final paragraphs of Section 2, noting only that the $\alpha_{\mathbf{i}}$, for which $i_{m}=0$ for some $m$ with $1 \leq m \leq r$, are in this instance already fixed. This completes the proof of the main conclusion of Theorem 2, the estimate (1.7) following directly.

\section{References}

[1] J. W. S. Cassels and R. C. Vaughan, Obituary: Ivan Matveevich Vinogradov, Bull. London Math. Soc. 17 (1985), 584-600; see Biogr. Mem. Fellows Royal Society 31 (1985), 613-631.

[2] G. H. Hardy and E. M. Wright, An Introduction to the Theory of Numbers, 5th ed., 4th reprint, Clarendon Press, Oxford, 1989.

[3] L.-K. Hua, Additive Theory of Prime Numbers, Amer. Math. Soc., Providence, 1965.

[4] N. N. Rogovskaya, An asymptotic formula for the number of solutions of a system of equations, in: Diophantine Approximations, Part II, Moskov. Gos. Univ., Moscow, 1986, 78-84 (in Russian).

[5] R. C. Vaughan and T. D. Wooley, On a certain nonary cubic form and related equations, Duke Math. J. 80 (1995), 669-735.

[6] I. M. Vinogradov, Selected Works, Springer, Berlin, 1985. 
[7] T. D. Wooley, Quasi-diagonal behaviour in certain mean value theorems of additive number theory, J. Amer. Math. Soc. 7 (1994), 221-245.

Mathematics Department

Huxley Building

Imperial College

180 Queen's Gate

London, SW7 2BZ, U.K.

E-mail: rvaughan@ma.ic.ac.uk
Mathematics Department

University of Michigan Ann Arbor, Michigan 48109-1003

U.S.A.

E-mail:wooley@math.lsa.umich.edu 